\title{
Distribution et statut de conservation du colobe de Geoffroy (Colobus vellerosus) au Bénin
}

\author{
S. DJEGO-DJOSSOU ${ }^{1 *}$ et B. SINSIN ${ }^{1}$ \\ Laboratoire d'Ecologie Appliquée, FSA /UAC, Bénin. \\ *Auteur correspondant, E-mail : djegosyl@yahoo.fr, Tél et Fax: +22930 308401 BP 526.
}

RÉSUMÉ

La présente étude s'est déroulée dans les formations végétales du Bénin échantillonnées dans les aires d'occurrence probables du colobe de Geoffroy (Colobus vellerosus). Des données de chorologie spatiale et d'identification des pratiques endogènes de conservation ont été collectées aux moyens des entretiens avec les populations, puis des prospections pédestres dans les aires protégées et forêts villageoises. Le colobe de Geoffroy est une espèce sociable de mœurs diurnes, inféodé aux formations forestières. Au Bénin, l'aire de répartition de l'espèce, évaluée à $9.000 \mathrm{~km}^{2}$ s'étend de la forêt classée de la Lama $\left(6^{\circ} 50^{\prime}-7^{\circ} 05^{\prime} \mathrm{N}\right.$ et $2^{\circ}$ $\left.2^{\circ} 15 \mathrm{E}\right)$ jusqu'à celle de $1^{\prime}$ Ouémé Supérieur $\left(9^{\circ} 15-9^{\circ} 45^{\prime} \mathrm{N}\right.$ et $\left.2^{\circ}-2^{\circ} 30 \mathrm{E}\right)$. La dynamique de cette répartition montre une régression de l'espèce corrélativement à une diminution et à une fragmentation de son aire dont la superficie est passée de $56.000 \mathrm{~km}^{2}$ en 1953 à $9.000 \mathrm{~km}^{2}$ de nos jours. Heureusement, certaines populations riveraines observent des pratiques ancestrales liées à la culture et à la religion en faveur de la conservation du colobe de Geoffroy.

(C) 2009 International Formulae Group. All rights reserved.

Mots clés : Colobe de Geoffroy, distribution, conservation, régression, Bénin.

\section{INTRODUCTION}

Les primates non-humains constituent une composante importante de la biodiversité grâce à leur rôle dans le maintien des écosystèmes forestiers. De par ce rôle écologique, ils contribuent largement à la dispersion des graines et à la régénération des arbres par zoochorie (Lambert, 2001 ; Koné et al., 2008 ). Appartenant à la famille des Cercopithecidés et la sous-famille des Colobinés, les colobes sont des singes africains apparus il y a 9 millions d'années (Karen, 1999). Au Bénin, parmi la dizaine de taxa de primates non-humains rencontrés, se trouvent deux espèces de colobe : le colobe olive et le colobe de Geoffroy, objet de la présente étude.

$\mathrm{Au}$ plan international, nombreuses sont les études réalisées sur les colobes au nombre desquelles on peut citer les multiples travaux de Oates (1985, 1994, 1996), Galat et GalatLuong (1982, 1983), Gautier et Gautier-Hion (1983, 1988, 1997), Oates et Trocco (1983), Karen (1999), Koné (2004), Bitty (2000), Korstenjs (2001) et Galat et Galat-Luong (2006). Toutes ces études ont abordé plusieurs aspects de la biologie, de l'écologie et de l'éthologie des colobes sans toutefois insister sur leur distribution géographique. D'un autre côté, les travaux de Kingdon (1980), Colyn (1988, 1991, 1993), Grubb (1990) et Colyn et al. (1991) ayant abordé l'aspect de 
distribution, restent encore focalisés sur l'importance des barrières géographiques puis des refuges dans la répartition des primates forestiers en général.

Au plan national (Bénin), les travaux réalisés sur les primates sont ceux de Sayer et Green (1984), Kafichoni (1987), Hanon (2001), Nobimè (2002), Teichroeb et al. (2001), Assogbadjo et Sinsin (2002), Sinsin et al. (2002), Campbell et al. (2007) et Nobimè et al. (2008). La plupart de ces études sont réalisées sur l'ensemble des primates et les différents résultats ont toujours montré que le colobe de Geoffroy est très peu fréquent dans les formations végétales avec des effectifs faibles. Ces paramètres inquiétants obligent à identifier tous les sites pouvant abriter l'espèce pour des études ultérieures en faveur de sa conservation. Heureusement, des croyances traditionnelles ont perduré dans certaines localités en faveur de la conservation locale du colobe de Geoffroy.

La présente étude qui aborde de façon spécifique le colobe de Geoffroy (Colobus vellerosus), a pour objectif principal d'établir les distributions ancienne et actuelle de l'espèce et d'identifier les mesures endogènes en faveur de sa conservation.

\section{MATERIEL ET METHODES \\ Milieu d'étude}

La présente étude s'est déroulée dans plusieurs aires protégées et zones libres appartenant aux aires d'occurrence probables $\mathrm{du}$ colobe de Geoffroy (Colobus vellerosus) au Bénin et réparties en zones guinéocongolaise, soudano-guinéenne puis soudanienne. Les aires protégées sont des milieux soumis à des mesures de protection juridique ou autres contrairement aux zones libres qui ne sont soumises à aucune forme de protection. La carte ci-dessous fait le point des zones étudiées (Figure 1).

\section{Méthodologie}

Les données ont été collectées grâce à des enquêtes exploratoires et rétrospectives, puis à des prospections pédestres dans les forêts végétales où la présence de l'espèce a été préalablement signalée par les enquêtés.
En effet, les enquêtes ont été surtout orientées vers les chasseurs, les gardes forestiers, les forestiers et toute personne ressource (ayant une bonne connaissance des primates et/ou capable d'imiter les cris des singes) retenus dans 41 villages. Un échantillonnage de 410 personnes au total à raison de 10 par village a été soumis à un questionnaire. Des planches portant diverses images de primates rencontrées au Bénin furent exploitées pour faciliter l'identification.

Les prospections ont lieu très tôt les matins lorsque la présence de l'espèce est préalablement signalée par les enquêtés. Au total, les forêts classées de la Lama, de l'Ouémé Boukou, de l'Ouémé Supérieur, de Wari- Maro, des Monts Kouffé, de DogoKétou, de Pénéssoulou, la forêt marécageuse de Lokoli, la forêt communautaire de Gnahouizoumê et la forêt sacrée de Kikélé ont fait l'objet de prospections. Dans la forêt de la Lama et de Pénéssoulou, les layons étaient suivis alors que dans la forêt marécageuse de Lokoli et à Gnanhouizoumê, les prospections ont été faites à pirogue et les groupes étaient identifiés selon les itinéraires favorables aux déplacements. Par contre, dans les forêts classées de l'Ouémé Boukou, de l'Ouémé Supérieur, des Monts Kouffé et de WariMaro, les données ont été collectées le long des transects. Par ailleurs, la forêt sacrée de Kikélé est un îlot forestier de 13 ha qui s'est retrouvé à l'intérieur du village de Kikélé et qui abrite une petite population de colobe de Geoffroy confiné dans la forêt dense sèche. C'est le seul endroit où l'espèce est observée facilement en milieu naturel.

$\mathrm{Au}$ cours des différentes prospections, les coordonnées géographiques des points de contacts étaient prises au GPS, de même que la taille des différents groupes. Les données géographiques ainsi collectées ont servi à la réalisation des cartes délimitant les aires de distribution passées et actuelle du colobe de Geoffroy. Dans les modèles de distribution réalisés, les contacts auditifs basés sur des vocalisations ont été considérés au même titre que les contacts visuels. Enfin, une approche ethnozoologique fondée sur l'identification et le respect de certaines pratiques ancestrales 


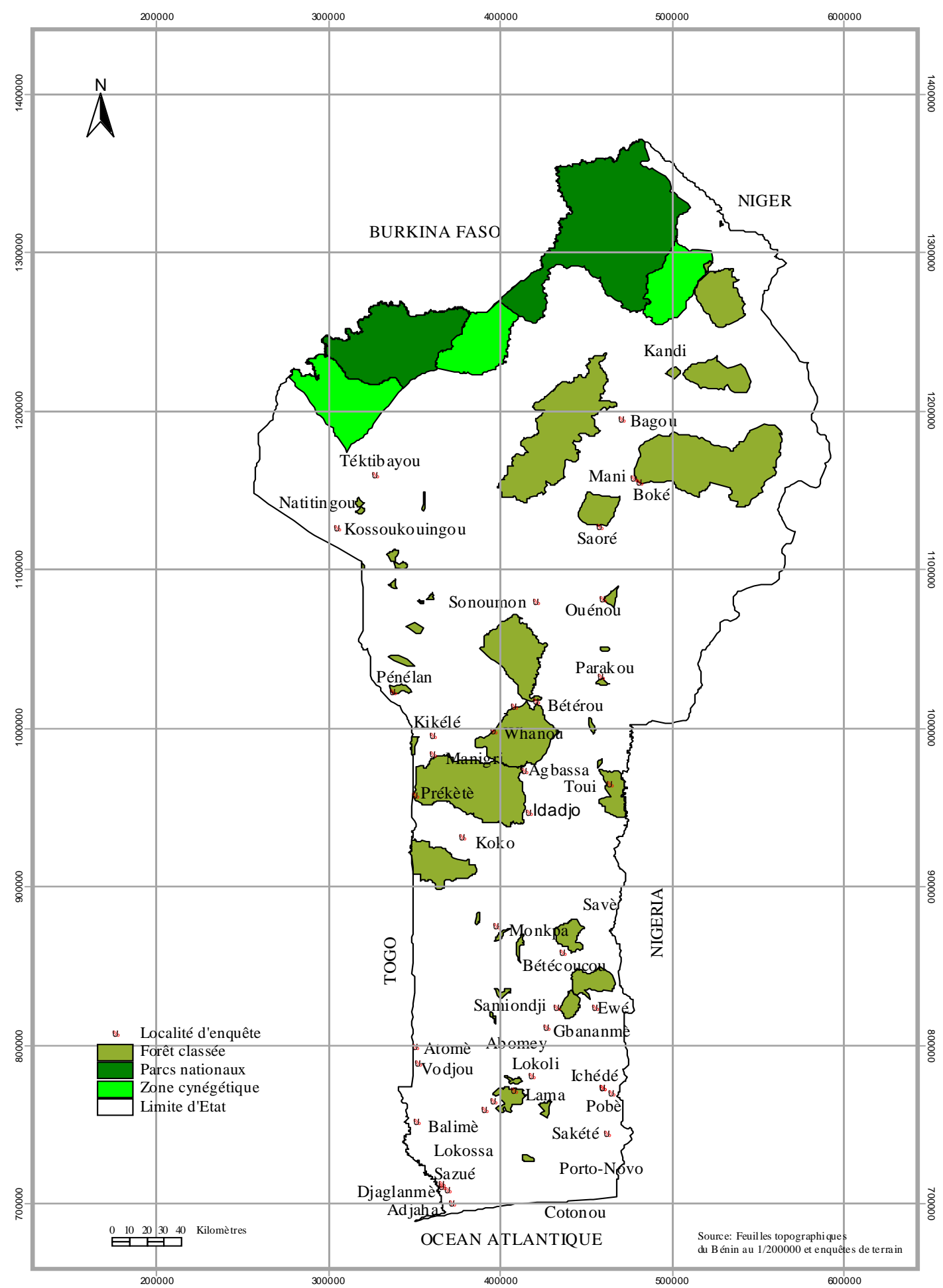

Figure 1: Situation géographique des villages enquêtés et des formations prospectées. 
s'est effectuée à partir des entretiens ouverts avec les populations riveraines et surtout les personnes âgées (au moins 30 ans).

\section{RESULTATS}

Distributions ancienne et actuelle du colobe de Geoffroy (Colobus vellerosus)

Sur l'ensemble des forêts prospectées, c'est seulement dans la forêt classée de DogoKétou que la présence du colobe de Geoffroy signalée par les populations n'a pas été confirmée. Dans toutes les autres formations, l'espèce était présente (contacts visuels et/ou auditifs). Les informations recueillies et les données collectées lors des enquêtes et prospections ont permis de réaliser les cartes et de dire qu'au cours de la période 19531983, l'aire de répartition du colobe de Geoffroy était continue et s'étendait entre les latitudes $\quad 6^{\circ} 55^{\prime} \mathrm{N}-11^{\circ} 15^{\prime} \mathrm{N} \quad$ regroupant plusieurs forêts classées et plusieurs zones libres avec une superficie totale estimée à $56.000 \mathrm{~km}^{2}$ (Figure 2).

Entre 1984 et 1999, on assiste à une fragmentation du bloc en trois sous-unités (Figure 3).

L'ensemble de trois sous-unités forme l'aire de répartition du colobe de Geoffroy avec une superficie estimée à $15.000 \mathrm{~km}^{2}$.

A présent, le colobe de Geoffroy est réparti entre les latitudes $6^{\circ} 55^{\prime} \mathrm{N}$ et $9^{\circ} 45^{\prime} \mathrm{N}$ avec trois sous-unités (Figure 4) et couvrant une superficie totale de $9.000 \mathrm{~km}^{2}$.

- La sous-unité située au nord regroupe les forêts classées des Monts Kouffé, de Warimaro, de Pénéssoulou, de l'Ouémé Supérieur, la région de Bassila avec la forêt sacrée de Kikélé ;

- la sous-unité médiane, réduite à la forêt classée de l'Ouémé Boukou ;

- la sous-unité inférieure qui comprend la forêt classée de la Lama, la vallée de l'Ouémé et la forêt marécageuse de Lokoli.

L'aire de distribution du colobe de Geoffroy a donc énormément régressé dans le temps comme en témoigne l'évolution des superficies qui passe de 56.000 à $9.000 \mathrm{~km}^{2}$ (Figure 4).
En une cinquantaine d'années, on assiste à une diminution de plus de $80 \%$. L'espèce subit une forte régression corrélativement à une réduction hyperbolique de son aire (Figure 5). La forte valeur du coefficient de corrélation $(-0,995)$ explique l'évolution en sens inverse des variables superficie et temps.

Le Tableau 1 fait le point des données collectées dans les différentes formations et les périodes où ces prospections ont eu lieu.

\section{Statut de conservation}

Dans le centre Bénin et en particulier dans la région de Bassila, le colobe de Geoffroy, à l'instar des autres singes, représente un tabou pour les populations locales qui ne doivent, ni consommer la viande, ni les pourchasser. La forêt sacrée de Kikélé située à $7 \mathrm{~km}$ de Bassila abrite, une petite population de colobe de Geoffroy dont l'effectif est estimé à 18 individus. Selon les investigations (Toko et Nobimè, 1997) et (Djègo-Djossou, 2003), les colobes seraient ramenés d'une grande forêt par les aïeux Tchabi Ota venus du Togo s'installer à Kikélé. Ayant bénéficié très tôt de l'assistance des hôtes protecteurs, des relations pacifiques se sont vite établies entre colobes et populations villageoises. Ces considérations sociologiques ont gagné très tôt tout le village qui considère le colobe comme une espèce sacrée. En plus de son caractère sacré, le colobe de Geoffroy bénéficie aussi d'un rôle cultuel. En effet, l'espèce incarne la pureté pour les populations musulmanes grâce à son corps noir et son visage entouré d'une crinière blanche à l'instar des responsables de culte musulman. De plus, il occupe la canopée des grands arbres et adopte une position tranquille comme dans une méditation profonde. Ces croyances traditionnelles ont sans doute contribué à la survie de l'espèce. En effet, les populations locales attachent plus du prix aux valeurs sacrées qu'aux valeurs juridiques. Enfin, nombreuses sont les populations qui ont souhaité la réintroduction de l'espèce là où elle a disparu grâce à son caractère sacré, son rôle cultuel mais aussi 


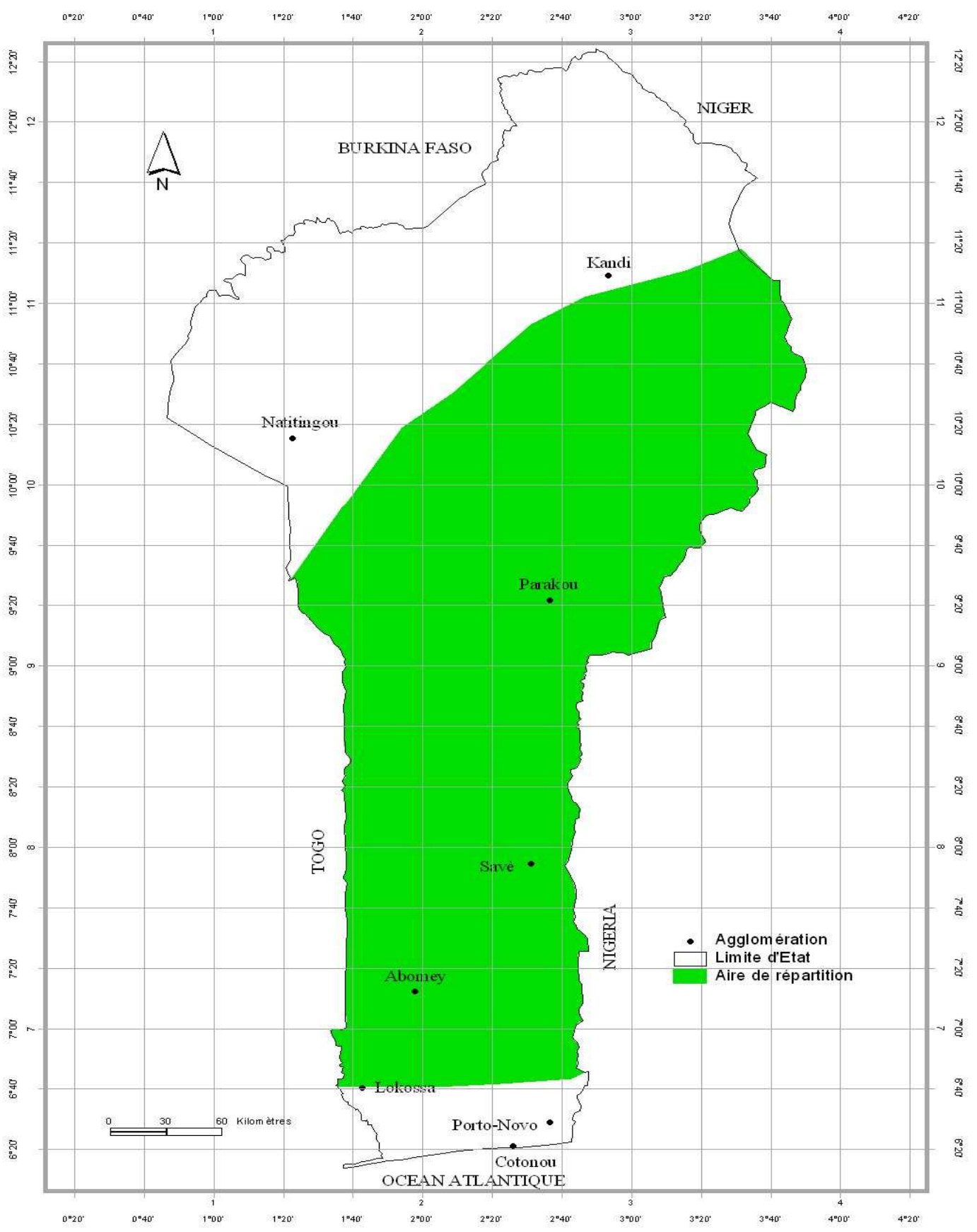

Figure 2: Répartition probable entre 1953 et 1983. 
S. DJEGO-DJOSSOU et B. SINSIN / Int. J. Biol. Chem. Sci. 3(6): 1386-1397, 2009

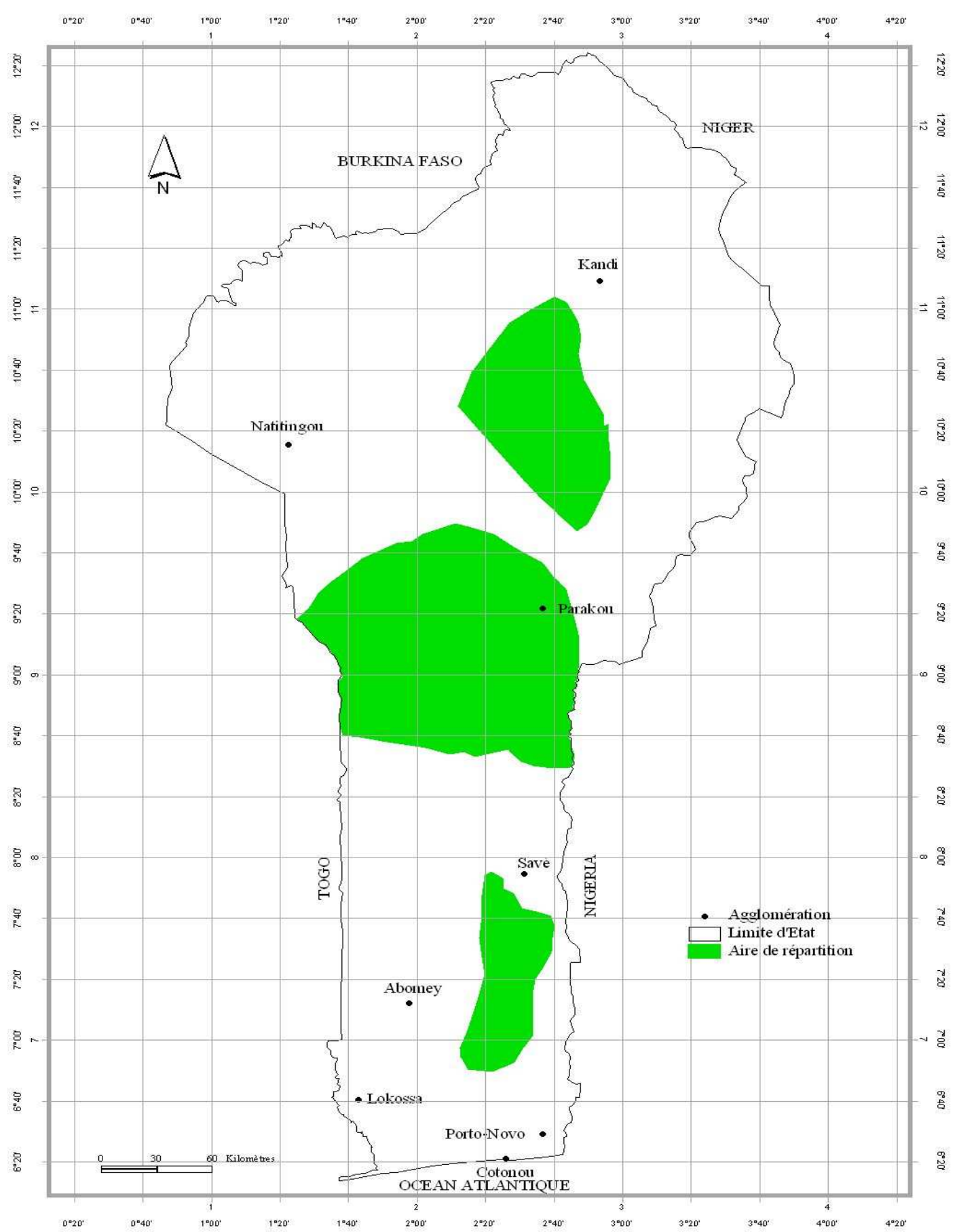

Figure 3: Répartition probable entre 1984 et 1999. 
S. DJEGO-DJOSSOU et B. SINSIN / Int. J. Biol. Chem. Sci. 3(6): 1386-1397, 2009

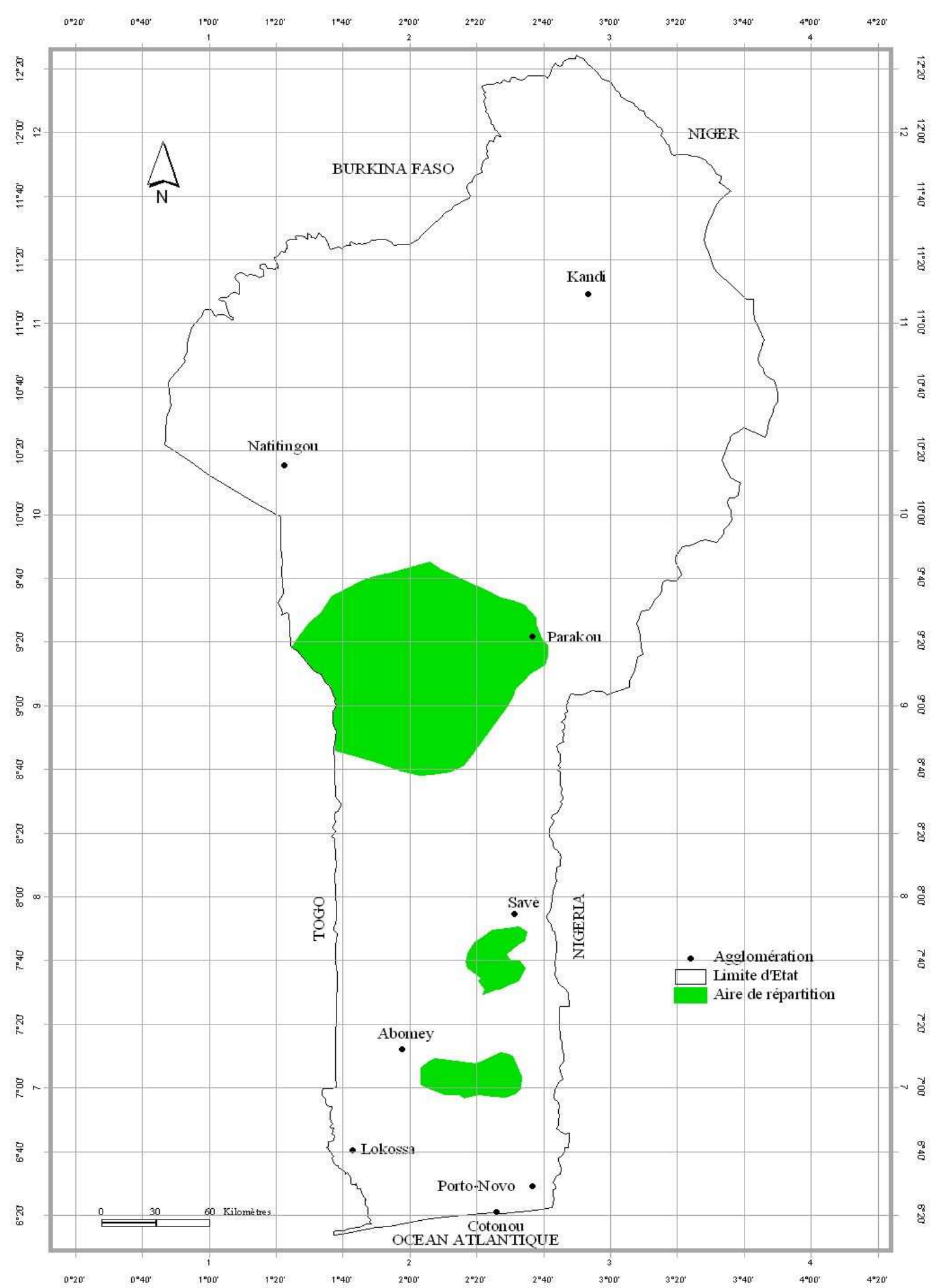

Figure 4: Répartition actuelle du colobe de Geoffroy.

1392 


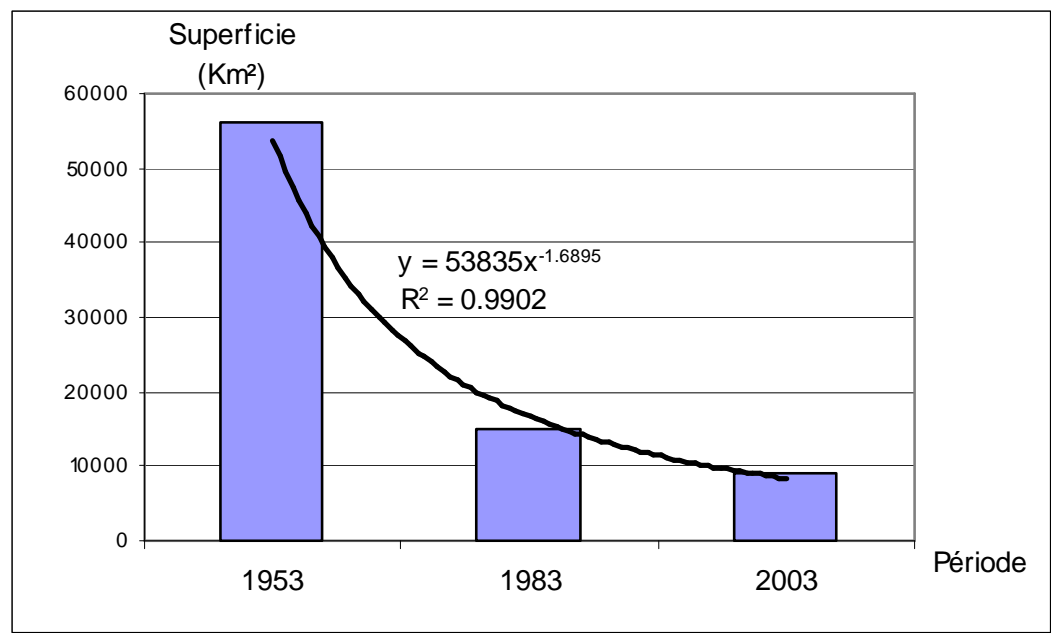

Figure 5 : Evolution de la superficie en fonction du temps.

grâce à son rôle dans les processus de maintien de la biodiversité forestier.

\section{DISCUSSION}

Distribution du colobe de Geoffroy

A l'origine, la distribution du colobe de Geoffroy était continue et les différentes populations pouvaient facilement échanger des flux de gènes. Une telle distribution avait caractérisé la plupart des espèces car la dégradation des forêts était moins alarmante. Actuellement, le colobe de Geoffroy présente une distribution plus ou moins large car celleci recouvre les zones guinéeo-congoloaise et guinéo-soudanienne. Toutefois, cette distribution reste discontinue à cause de la fragmentation de son habitat. Celle-ci a pour conséquence, la diminution spectaculaire de la superficie de l'aire de répartition qui passe de 56.000 à $9.000 \mathrm{~km}^{2}$. Face à ce résultat, le colobe de Geoffroy pourrait disparaître dans un proche avenir si aucune action n'est entreprise. Cela justifie le besoin grandissant de conservation de l'espèce et l'intérêt qu'il faut lui accorder dans le contexte de la conservation de la biodiversité. Campbell en 2005 avait obtenu une distribution similaire lors de ses prospections. Dans cette fragmentation de l'habitat, on assiste à une concentration des tâches dans la partie méridionale. L'espèce a trouvé refuge dans les zones où se superposent un ensemble de facteurs favorables à sa survie.

Cette distribution discontinue qui caractérise la population du colobe de Geoffroy, s'observe aussi avec le singe à ventre roux Cercopithecus e. erythrogaster au Bénin (Sinsin et al., 2002), tout comme Erythrocebus patas, Papio anubis. Par contre Cercopithecus mona et Chlorocebus aethiops présentent une large distribution (Sayer et Green, 1984). Pendant longtemps, le colobe de Geoffroy a payé un lourd tribut à la chasse (Gautier-Hion et al., 1999) grâce à la valeur marchande de sa fourrure, utilisée dans la confection des chaussures locales et des tambours. De plus, le braconnage pour la recherche de sa viande a perturbé les différentes populations. Ces facteurs ont probablement conduit à une diminution drastique des effectifs du colobe de Geoffroy dans les diverses formations.

Par ailleurs, les paramètres d'inventaire sont faibles car la période d'étude relativement courte, correspond à la saison pluvieuse où les contacts sont faibles à cause de la densité de la végétation et du fait que les animaux se déplacent peu. De plus, loin d'être un inventaire exhaustif, l'étude a pour but principal de savoir si les formations végétales abritant ou non le colobe de Geoffroy. Les effectifs ont été donc obtenus en faisant une 
Tableau 1 : Différentes forêts prospectées et résultats des prospections.

\begin{tabular}{|c|c|c|c|c|c|c|c|}
\hline Forêts prospectées & $\begin{array}{l}\text { Superficie } \\
\text { (ha) }\end{array}$ & $\begin{array}{c}\text { Présence ou absence } \\
\text { de l'espèce }\end{array}$ & $\begin{array}{c}\text { Période de } \\
\text { prospections }\end{array}$ & $\begin{array}{c}\text { Nombre de } \\
\text { contacts visuels }\end{array}$ & $\begin{array}{c}\text { Nombre de } \\
\text { contacts auditifs }\end{array}$ & $\begin{array}{c}\text { Fréquence } \\
(\%)\end{array}$ & $\begin{array}{c}\text { Effectif } \\
\text { total }\end{array}$ \\
\hline Forêt classée de Lama & 16250 & Présence (vu et entendu) & mai & 6 & 3 & 7 & 27 \\
\hline Forêt classée de Pénéssoulou & 5470 & Présence (vu et entendu) & juillet & 4 & 5 & 5 & 21 \\
\hline Forêt classée des Monts Kouffé & 180300 & Présence (entendu) & août & 0 & 3 & 5 & indéterminé \\
\hline Forêt classée de Wari-Maro & 107500 & Présence (entendu) & août & 0 & 2 & 6 & indéterminé \\
\hline Forêt marécageuse de Lokoli & 500 & Présence (vu et entendu) & mai & 3 & 3 & 4 & 17 \\
\hline $\begin{array}{l}\text { Forêt communautaire de } \\
\text { Gnanhouizounmê (vallée de } \\
\text { l'ouémé) }\end{array}$ & 190 & Présence (vu et entendu) & mi-avril & 2 & 2 & 6 & 9 \\
\hline $\begin{array}{l}\text { Forêt classée de l'Ouémé } \\
\text { Supérieur }\end{array}$ & 107542 & Présence (entendu) & juillet & 0 & 2 & 4 & indéterminé \\
\hline Forêt classée de Dogo-Kétou & 50000 & Absence & juin & 0 & 0 & 0 & 0 \\
\hline Forêt classée de l'Ouémé Boukou & 20500 & Présence (entendu) & juin & 0 & 3 & 4 & indéterminé \\
\hline Forêt sacrée de Kikélé & 13 & Présence (vu et entendu) & juillet & $\begin{array}{l}\text { Population } \\
\text { territoriale }\end{array}$ & $\begin{array}{l}\text { Population } \\
\text { territoriale }\end{array}$ & 100 & 18 \\
\hline
\end{tabular}


simple sommation des effectifs des différents groupes. Ces effectifs obtenus sont tous sousestimés car seuls les contacts visuels étaient pris en compte. Les observations ont montré le colobe en groupes monospécifiques mais aussi en groupes plurispécifiques. Dans la forêt classée de la Lama, le colobe de Geoffroy s'associe facilement avec le Mone Cercopithecus mona (Nobimè, 2002). Dans la plupart des formations, le Mone était l'espèce la plus fréquente, ce qui justifie son caractère commun. Le colobe de Geoffroy quant à lui reste confiné en nombre très réduit sur une petite superficie, il se déplace peu et est beaucoup plus discret (Assogbadjo et Sinsin, 2002).

La superficie actuelle de l'aire de répartition discontinue liée aux effectifs faibles restent préjudiciables à la survie à long terme du colobe et accentue la vulnérabilité de l'espèce. En effet, l'isolement des différentes populations pourrait occasionner des risques de consanguinité et d'appauvrissement génétique.

\section{Pratiques ancestrales liées à la conservation de l'espèce}

Le colobe de Geoffroy, tel qu'il est conservé localement à kikélé, est aussi vénéré au Ghana. De telles pratiques ancestrales de conservation sont connues dans la région de Brong Ahafo au Ghana (Fiema et Boabeng) où le colobe de Geoffroy, le cercopithèque mone ainsi que leurs habitats dans la forêt décidue sont depuis longtemps protégées par des croyances et les traditions locales (Saj et al. 2006, Campbell et al., 2008). Dans cette région, quand un singe meurt, il est pleuré et enterré comme un être humain (Akowuah, 1975). Ces mesures de conservation endogènes du colobe de Geoffroy développées à Kikélé sont donc à promouvoir car elles assurent la sécurité des lieux et la quiétude, facteurs de retour de la faune. Déjà à Kikélé, des groupes de Cercopithecus mona fréquentent l'habitat du colobe de Geoffroy.

\section{Conclusion}

Le Bénin abrite une population de colobe de Geoffroy (Colobus vellerosus) dont l'aire actuelle est fragmentée et s'étend entre les latitudes $6^{\circ} 55^{\prime} \mathrm{N}$ et $9^{\circ} 45^{\prime} \mathrm{N}$ avec une concentration des groupes dans la région méridionale (région des Monts Kouffé). Dans cette région, des pratiques ancestrales liées aux caractères sacré et cultuel de l'espèce constituent de véritables mesures de conservation endogènes. Malgré leur efficacité, ces mesures ne permettent pas à elles seules d'assurer la survie à long terme de la population qui d'ailleurs est isolée sur une île écologique. Elles doivent être renforcées par l'élaboration d'un véritable plan de conservation pour la protection à long terme de l'espèce. Il est donc nécessaire de poursuivre les prospections pour préciser non seulement l'aire d'occupation de l'espèce mais aussi d'effectuer des inventaires pour apprécier les populations de colobe.

\section{RÉFÉRENCES BIBLIOGRAPHIQUES}

Akowuah DK, Rice K, Merz A, Sackey VA. 1975. The children of gods. J. Ghana Wildlife Soc., 1(2): 19-22.

Assogbadjo A, Sinsin B. 2002. Diversité, structure et comportement des primates de la forêt marécageuse de Lokoli au Bénin. Biogeographica, 78(4): 129-140.

Bitty EA. 2000. Le déterminisme de l'utilisation du domaine vital du colobe blanc noir Colobus polykomos polykomos (Zimmerman, 1780) dans le parc national de Taï.

Campbell G. 2005. Distribution, Census and Habitat Preferences of Primate Species in the Dahomey Gap (West Africa), with Particular Emphasis on the Red-bellied Guenon (Cercopithecus erythrogaster erythrogaster). Thesis of Master; University of Calgary, P.114.

Campbell G, Teichroeb J, Paterson JD. 2008. Distribution of diurnal primates species in Togo and Benin. Folia Primatologica 79(1):15-30. 
Colyn M. 1987. Les primates des forêts ombrophiles de la cuvette du Zaïre: interprétations zoogéographiques des modèles de distribution. Rev. Zool. Afr., 10(1): 183-196.

Colyn M. 1991. L’importance géographique du bassin du fleuve de Zaïre pour la spéciation: le cas des primates simiens. Ann. Musée Roy. Afr. Cent. Tervuren, 264.

Colyn M, Gauthier-Hion A, Verheyen W. 1991. A reappraisal of palaoenvironnemental history in Central Africa: evidence for a major fluvial refuge in the Zaïre basin. J. Biogeogr., 16: 403-407.

Colyn M. 1993. Coat color polymorphysm of red colobus monkeys (Colobus badius, Primates Colobinae) in Eastern Zaïre: Taxonomic and Biogeographic implications. J. Afr. Zool., 107: 301-320.

Djègo-Djossou S. 2003. Détermination de l'aire de répartition de la population de colobe de Geoffoy Colobus vellerosus et statuts de conservation au Bénin, P 97.

Galat-Luong A, Galat G. 1982. Abondance relative et associations plurispécifiques des primates diurnes du P.N.T., Côted'Ivoire. Rapport du centre O.R.S.T.O.M. d'Adiopodoumé, Abidjan, $39 \mathrm{p}$.

Galat-Luong A, Galat G. 1982. Domaines vitaux, densités et stratification de sept Cercopithecidés forestiers d'un même site du Parc National de Taï, Côted'Ivoire: premiers résultats. Rapport du centre O.R.S.T.O.M. d'Adiopodoumé, Abidjan.

Galat-Luong A. 1983. Socio-écologie de trois colobes sympatriques, Colobus badius, C. polykomos et $\mathrm{C}$. verus du parc National de Taï, Côte d'Ivoire. Thèse de doctorat d'Université, Université Pierre et Marie Curie, Paris, pp 226.

Galat-Luong A, Galat G. 2006. Résistance pugnance des colobes bai du Saloum. Journal de l'IRD.

Gautier-Hion A, Quris R, Gautier JP. 1983. Monospecific vs polyspecific life: a comparative study of foraging and antipredatory tactics in a community of Cercopithecus monkeys. Behav. Ecol. Sociobiol., 12: 325-335.

Gautier-Hion A, Tutin CEG. 1988. Simultaneous attack by adult males of apolyspecific troop of monkeys against a crowned hawk eagle. Folia Primatol., 51: 149-151.

Gautier-Hion A, Gautier J-P, Moungazin A. 1997. Do black Colobus in mixedspecies groups benefit from increased foraging, strategy. Compte Rendu de l'Académie des Sciences, 320: 87-71.

Gautier-Hion A, Colyn M, Gautier JP. 1999. Histoire Naturelle des Primates d'Afrique Centrale. Ecofac, Multipress: Gabon ; 141.

Grubb P. 1990. Primates geographic in the Afro-tropical forest biome. In Vertebrates in Tropics, Peters G, Hutterer R (eds). Museum Alexander Koening : Bonn ; 187-214.

Hanon L. 2001. La végétation et le terroir de Togbota Agué (Sud-Bénin). Analyse des possibiltés et obstacles à la conservation du singe à ventre rouge, Cercopithecus erythrogaster erythrogaster Gray Mémoire de fin d'études en vue de l'obtention du grade d'ingénieur Agronome. Université Libres de Bruxelles, Belgique, p 81.

Kafichoni B. 1987. Etude écoéthologique d'un primate, Cercopithecus aethiops tantalus, dans la zone sud de la forêt classée de la Lama. CPU/ UAC, Bénin.

Karen B. 1999. Primate behavioral ecology. USA p.392

Kingdon J. 1980. The roles of visual signals and face patterns in African forests monkeys of the genus Cercopithecus. Trans. Zool. Soc. Lond., 35: 425-475.

Koné I. 2004. Effet du braconnage sur quelques aspects du comportement du colobe bai-Procolobus [Piliocolobus] badius (Kerr)- et du Cercopithèque Diane- Cercopithecus diana diana (L.) dans le parc National de Taï, Côte d'Ivoire. 
Koné I, Lambert EJ, Refisch J, Bakayoko A. 2008. Primate seed dispersal and its potential role in maintaining useful tree species in the Taï region, Côte d'Ivoire: implications for the conservation of forest fragments. Tropical Conservation Science, 1(3): 293-306.

Korstjens AH. 2001. An analysis of the socio-ecological strategies of the three Colobines of Taï Ph.D, University of Ivory Coast,. $173 \mathrm{p}$.

Lambert JE. 2001. Reid-Tailed Guenons (Cercopithecus ascanius) and Strychnos mitis: Evidence for plant Benefits Beyond seed Dispersal. Int. J. Primatol, 22(2): 189.

Nobimè G, Toko I. 1997. Genre de vie des colobes blancs et noirs de Kikélé et des primates de la forêt classée de Pénéssoulou (Sous-préfecture de Bassila, nord- Bénin). Rapport $\mathrm{N}^{\circ} 1$ d'activité. LEA/UAC.

Nobimè G. 2002. Collecte de données de base pour la protection du singe à ventre rouge (Cercopithecus erythrogaster) dans la forêt classée de la Lama au Bénin. Mémoire de DEA/FLASH. Ecole Doctorale Pluridisciplinaire Espaces, Cultures et développement, Bénin P 75.

Nobimè G, Sinsin B, Lernould JM. 2009. Ecological factors determining the distribution of red-bellied guenon Cercopithecus e. erythrogaster in Bénin and Togo. Int. J. Biol. Chem. Sci., 3(3): 606-611.
Oates JF, Trocco TF. 1983. Taxonomy and phylogeny of black- and- white colobus monkey. Folia Primatologina, 40: 83113.

Oates JF. 1985. Action plan for African Primate Conservation. UICN/SSC Primate Specialist Group. UICN, Gland, Suisse.

Oates J. 1994. The natural history of African Colobines. In Colobine Monkey: their Ecology, Behaviour and Evolution, Davies AG, Oates JF (eds). Cambridge University Press; 75-128.

Oates J. 1996. African Primates: Status and Conservation Action Plan (Revised edition). The World Conservation Union (UICN): Gland, Switzerland; 80.

Saj TL, Mather C, Sicotte P. 2006. Traditional taboos in biological conservation: the case of Colobus vellerosus at the Boabeng-Fiema Monkey Sanctuary, Ghana Central. Social Science Information, 2: 285- 310.

Sayer JA, Green A. 1984. The distribution and status of large mammals in Benin. Mammal Rev., 14(1): 37-50.

Sinsin B, Nobimè G, Téhou A, Bekhuis P, Tchibozo S. 2002. Past and present distribution of red-bellied monkey Cercopithecus erythrogaster erythrogaster Gray in Bénin. Folia Primatologica, 73(2-3): 116-123.

Teichroeb JA, Saj TL, Paterson JD, Sicotte P. 2001. Effect of Group Size on Activity Budgets in Colobus vellerosus in Ghana. International Journal of Primatology, 24: 743- 758. 